\title{
Mathematical Analysis of a Model of River Channel Formation
}

\author{
J. I. Díaz, ${ }^{1}$ A. C. Fowler, ${ }^{2}$ A. I. Muñoz, ${ }^{3}$ and E. Schiavi ${ }^{3}$
}

\begin{abstract}
The study of overland flow of water over an erodible sediment leads to a coupled model describing the evolution of the topographic elevation and the depth of the overland water film. The spatially uniform solution of this model is unstable, and this instability corresponds to the formation of rills, which in reality then grow and coalesce to form large-scale river channels. In this paper we consider the deduction and mathematical analysis of a deterministic model describing river channel formation and the evolution of its depth. The model involves a degenerate nonlinear parabolic equation (satisfied on the interior of the support of the solution) with a super-linear source term and a prescribed constant mass. We propose here a global formulation of the problem (formulated in the whole space, beyond the support of the solution) which allows us to show the existence of a solution and leads to a suitable numerical scheme for its approximation. A particular novelty of the model is that the evolving channel self-determines its own width, without the need to pose any extra conditions at the channel margin.
\end{abstract}

Key words: River models, landscape evolution, nonlinear parabolic equations, free boundaries, singular free boundary flux.

\section{Introduction}

In a recent paper, FOwLER et al. (2007) addressed the question of how rivers form in the landscape. They derived a nonlinear partial differential equation of diffusive type to describe the depth of an evolving channel, and it is the mathematical analysis of this equation which forms the substantive part of the present paper. In this introduction we begin by indicating the physical context within which this equation arises, and we sketch the way in which it is derived.

It is a matter of common experience that rainfall on land surfaces does not drain uniformly. Even on short time scales, small-scale channels called rills form, and over longer time scales, these rills evolve and merge, forming progressively larger channels. At the same time, the flow in the developing channels erodes the hillslope, cutting its way down, and the overland flow into the channels causes sub-channels or tributaries to form,

${ }^{1}$ Departamento de Matemática Aplicada, Universidad Complutense de Madrid, 28040 Madrid, Spain. E-mail: ji_diaz@mat.ucm.es.

${ }^{2}$ MACSI, Department of Mathematics and Statistics, University of Limerick, Limerick, Ireland. E-mail: fowler@math.ox.ac.uk.

3 Departamento de Matemática Aplicada, E.S.C.E.T. Universidad Rey Juan Carlos, E 28933 Móstoles, Madrid, Spain. E-mail: anaisabel.munoz@urjc.es, emanuele.schiavi@urjc.es. 
so that in a mature landscape, such as that shown in Figure 1, a fractal-like pattern of river channels dissects the landscape.

The mathematical understanding of the drainage process on the large scale of a catchment is challenging. Just as for air transport in the lungs, or for liquid transport in crystallizing mushy zones, the medium may be best represented as a porous network, but (like the second example) it is one whose transport properties are formed by the transport process itself. We thus need to understand the mechanisms whereby channels form in the first place, and which govern their size and transport capacity.

The ingredients of a suitable model are variables describing water flow and sediment transport, and the mechanism of channel formation arises through an instability, in which locally increased flow causes increased erosion, which in turn increases the flow depth and thus also the flow. This positive feedback induces instability, as was shown by SмITH and BRETHERTON (1972), in their pioneering study.

Smith and Bretherton's study was later elaborated by LoEwENHERz (1991), and LoEWENHERZ-LAWRENCE (1994), who was particularly concerned with the issue of wavelength selection, something which also formed the principal concern of IzUMI and PARKER (1995, 2000). Nonlinear studies of channel development and topographic evolution focussed on catchment scale problems, such as that of WILLGOOSE et al. (1991), however such efforts were unable to compute the solution of the governing models directly, essentially because of the stiffness of the system. WiLlgoose et al. (1991) reverted to an artificial channel indicator variable, and KRAMER and MARDER (1992) used cellular lattice models, a development which has formed the thrust of simulation models since, e.g., those of HowARD (1994) and TUCKER and Slingerland (1994). There have been efforts to solve the Smith-Bretherton model directly (e.g., SMITH et al., 1997; BIRNIR et al., 2001), although these are problematical, unsurprisingly since the original Smith-Bretherton model is actually ill-posed.

The starting point for Smith and Bretherton's study was a coupled set of partial differential equations describing $s(x, y, t)$, the hill slope elevation, and $h(x, y, t)$, the water depth. The model takes the form

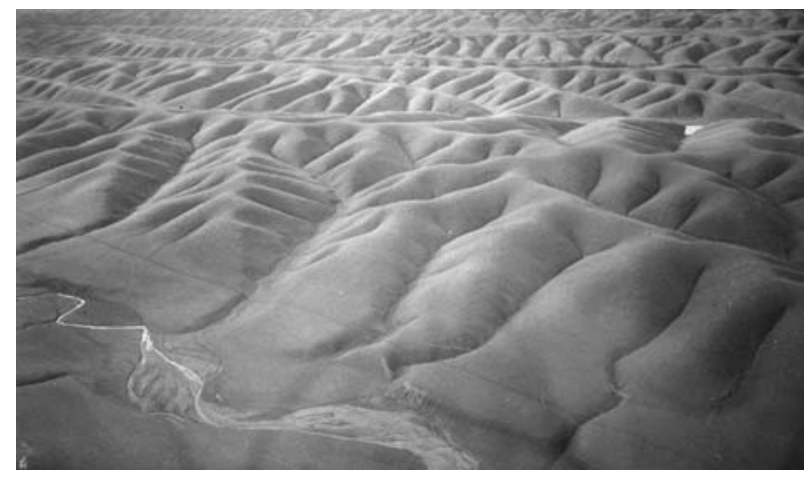

Figure 1

Hillslope topography. Photograph courtesy (Gary Parker). 


$$
\begin{gathered}
\nabla \cdot(h \mathbf{u})=r, \\
s_{t}+\nabla \cdot \mathbf{q}=U,
\end{gathered}
$$

and represents conservation of mass of water and sediment. The mean water velocity $\mathbf{u}$ is determined through a momentum balance equation, while the sediment flux $\mathbf{q}$ is usually taken as an empirically prescribed function of flow-induced bed stress and bed slope, the resulting combination (the effective bed stress) being denoted $\tau$. The source term $r$ represents rainfall, while $U$ represents tectonic uplift. The time derivative in the water mass equation is ignored, on the basis that the time scale for evolution of the hill slope is considerably longer than that for water flow.

In order to complete this model, we assume it has been written in dimensionless form, so that the variables are $O(1)$. One can show that suitable models for the flow speed $\mathbf{u}$ and effective bed stress $\tau$ are

$$
\begin{aligned}
& \mathbf{u}=h^{1 / 2}|\nabla \eta|^{1 / 2} \mathbf{n}, \\
& \tau=\mathbf{u}|\mathbf{u}|-\beta \nabla s,
\end{aligned}
$$

where typically $\beta=O(1)$, and the down-water slope normal $\mathbf{n}$ is defined by

$$
\mathbf{n}=-\frac{\nabla \eta}{|\nabla \eta|}
$$

$\eta$ represents the water surface elevation, and in dimensionless terms is related to hillslope elevation $s$ and water film thickness $h$ by

$$
\eta=s+\delta h .
$$

The parameter $\delta$ is very small, a typical estimate being $10^{-5}$. Finally, the sediment flux is taken to have the form

$$
\mathbf{q}=V(\tau) \mathbf{N},
$$

where $\tau=|\tau|$ and the down-sediment flow normal $\mathbf{N}$ is

$$
\mathbf{N}=\frac{\tau}{\tau} .
$$

$V$ is an increasing function of $\tau$, with $V \approx \tau^{3 / 2}$ being a popular choice (this essentially stemming from the model of MeYer-Peter and Müller, 1948).

This model admits steady solutions corresponding to overland flow, in which the variables $\eta=\eta_{0}(X)$ and $h=h_{0}(X)$ are functions only of the downslope coordinate $X$, and the flow of both water and sediment is in the $X$ direction, thus $\mathbf{n}=\mathbf{N}=\mathbf{i}$, the unit vector along the $X$ axis. The Smith-Bretherton analysis was based on taking the limit $\delta \rightarrow 0$, and in this case it is easy to see that always $\mathbf{n}=\mathbf{N}$, even for non-unidirectional flows. Smith and Bretherton then found instability to arise in an ill-posed way, through a negative effective diffusivity in the cross-stream $y$ direction. This leads to unbounded growth at small transverse wavelengths, but LOEWENHERZ-LAWRENCE (1994) was able to show that 
the instability was regularized at high wave number by including the small term $\delta$ in (1.4), which has the crucial effect of making $\mathbf{n} \neq \mathbf{N}$.

It is this observation which allows analytic progress in the model. Uniform overland flow is unable to $y$-dependent perturbations of small wavelength, and we can examine the nonlinear evolution of these by directly seeking asymptotic expansions in terms of $\delta$. To do so, we firstly suppose that the channels which form are aligned in the $X$ direction, and (sensibly) that the perturbation to the water surface is small, comparable to the overland flow depth:

$$
\eta=\eta_{0}+\delta Z
$$

We may then linearize the geometry of the system, to find that

$$
\begin{aligned}
& \mathbf{n}=\mathbf{i}-\frac{\delta Z_{y}}{S} \mathbf{j}+\cdots, \\
& \mathbf{N}=\mathbf{i}-\frac{\delta}{S}\left\{Z_{y}-\frac{\beta}{h+\beta} h_{y}\right\} \mathbf{j}+\cdots,
\end{aligned}
$$

where $\mathbf{j}$ is the unit vector in the $y$ direction and $S(X)=\left|\eta^{\prime}{ }_{0}(X)\right|$ is the unperturbed downhill slope.

The nonlinear channel evolution then arises from a rescaling of the hillslope evolution equation, in which we put

$$
y=\delta^{1 / 2} Y, \quad h=\frac{H}{\delta^{1 / 3}}, \quad t=\delta^{7 / 6} T
$$

after some algebra, we find the leading order sediment transport equation takes the form

$$
\frac{\partial H}{\partial T}=S^{\prime} S^{1 / 2} H^{3 / 2}+S^{1 / 2} \frac{\partial}{\partial Y}\left[\beta H^{1 / 2} \frac{\partial H}{\partial Y}\right],
$$

where $S^{\prime}=d S / d X$.

It is important to note that this equation arises through conservation of sediment. Only $Y$ derivatives are present, because the lateral length scale is so much smaller than the downslope one. The perturbation $Z$ to the water surface is in fact then determined by quadrature of the water conservation equation, but integration of this equation in the across stream direction yields the integral constraint

$$
\int_{-\infty}^{\infty} H^{3 / 2} d Y=\frac{2 \operatorname{Lr} X}{S^{1 / 2}}
$$

where $L$ is the spacing (on the original hillslope length scale for $y$ ) between channels; the limits in (1.11) are, however, infinite because the integral is with respect to the much smaller channel width length scale. Suitable initial and boundary conditions for the channel depth are that

$$
H \rightarrow 0 \quad \text { as } \quad Y \rightarrow \pm \infty, \quad H=H_{0}(Y) \quad \text { at } \quad T=0 .
$$


An alternative to the initial condition is to assume that $H \rightarrow 0$ as $T \rightarrow-\infty$, corresponding to the evolution of an initial infinitesimal perturbation. (1.12) mimics this if we suppose that $H_{0}$ is everywhere small in value.

The equation (1.10), together with the integral constraint (1.11) and initial/boundary conditions (1.12), form the basis of our study. We will assume that $S^{\prime}>0$, so that the nonlinear term in (1.10) is a source. Since the downslope coordinate only appears in the coefficients, it can be scaled out of the problem. Indeed, if we define

$$
H=\left(\frac{6}{\beta}\right)^{1 / 3}(\operatorname{Lr} X)^{2 / 3} u, \quad T=\left(\frac{\beta}{6}\right)^{1 / 6} \frac{t}{S^{1 / 2} S^{\prime}(\operatorname{Lr} X)^{1 / 3}}, \quad Y=\left(\frac{2 \beta}{3 S^{\prime}}\right)^{1 / 2} x
$$

(note that this definition of $t$ is distinct from that used previously), the problem to be studied can be written in the form

$$
\begin{gathered}
u_{t}=u^{3 / 2}+\left(u^{3 / 2}\right)_{x x}, \\
\int_{-\infty}^{\infty} u^{3 / 2} d x=1, \quad u \rightarrow 0 \quad \text { as } \quad x \rightarrow \pm \infty \\
u=u_{0}(x) \quad \text { at } \quad t=0 .
\end{gathered}
$$

\section{Mathematical Analysis}

We consider problem (1.14) assuming an initial thickness perturbation $u_{0}(x)$ satisfying some natural physically based hypothesis, i.e., a bounded and nonnegative function with a compact and connected support $\left[-\zeta_{0}, \zeta_{0}\right]$ such that $\int_{0}^{+\infty} u_{0}^{m}(x) d x=M / 2$, for $m>1$ (including the case of $m=3 / 2$ of (1.14)). For the sake of simplicity of the exposition we also assume symmetric initial data.

In the rest of the paper, we shall be especially interested in the question of global solvability (in time) of the following problem: Find a continuous curve $\zeta:[0,+\infty) \rightarrow \mathbb{R}^{+}$and a function $u: \mathcal{P} \rightarrow[0,+\infty)$ (regular enough) such that

$$
(S L) \begin{cases}u_{t}=\left(u^{m}\right)_{x x}+u^{m}, & \text { in } \mathcal{D}^{\prime}(\mathcal{P}), \\ u(x, 0)=u_{0}(x) & \text { a.e. } x \in \Omega_{0}, \\ u(x, t)>0, & \text { a.e. }(x, t) \in \mathcal{P}, \\ u(x, t) \equiv 0, & \text { a.e. }(x, t) \notin \mathcal{P}, \\ u(\zeta(t), t)=0, \quad\left(u^{m}\right)_{x}(0, t)=0 & \text { a.e. } t \in(0,+\infty), \\ \zeta(0)=\zeta_{0} \text { and } \zeta(t)>0 & \text { for any } t \geq 0, \\ \zeta(t) & \text { a.e. } t \in(0,+\infty) . \\ \int_{0}^{m} u^{m}(x, t) d x=\frac{M}{2} & \end{cases}
$$


where $\Omega_{0}=\left(0, \zeta_{0}\right), \Omega_{t}=(0, \zeta(t)) \times\{t\}, \mathcal{P}=\cup_{t>0} \Omega_{t}$. Notice that $\mathcal{D}^{\prime}(\mathcal{P})$ denotes the space of distributions on $\mathcal{P}$ and $\mathcal{P}$ is the positivity subset of the solution. Later on we shall make more precise the (minimal) regularity of the solution. The function $\zeta(t)$ is called the interface separating the (connected) region where $u(x, t)>0$ from the region where $u(x, t)=0$. It is unknown and it is usually called the free or moving boundary of the problem. Due to the free boundary, we shall refer to the strong formulation $(S L)$ as the strong-local formulation. We emphasize that the mass conservation constraint

$$
\int_{0}^{\zeta(t)} u^{m}(x, t) d x=\frac{M}{2} \quad \text { a.e. } t \in(0,+\infty)
$$

prevents possible blow-up phenomena which could arise (without this condition) due to the presence of the source term $u^{m}$ in the equation.

An important difficulty, in order to get a global formulation (i.e., extended to the entire domain $(x, t) \in(0,+\infty) \times(0,+\infty)$, and not only on $(x, t) \in \mathcal{P})$, is the necessity to provide a suitable description of the flux $-\left(u^{m}\right)_{x}(\zeta(t), t)$ at the free boundary. This leads to a new constrained global formulation suitable for mathematical analysis and numerical resolution. In the next subsections we propose the global formulation of the model for the stationary regime. The transient regime is dealt with in subsection 2.2. Finally, in subsection 2.3, a finite element method is described and numerical results are presented.

\subsection{The Stationary Case}

We first consider the strong formulation of the stationary problem associated to ( $S L$ ). Let $M$ be a positive, fixed, real number and define $v=u^{m}$. Then we look for a solution of

$$
-v_{x x}-v=0, \quad v^{\prime}(0)=0, \quad \lim _{x \rightarrow+\infty} v(x)=0, \quad \int_{0}^{+\infty}|v(x)| d x=\frac{M}{2} .
$$

We first observe that the formulation does not correspond to a standard constrained problem of the Calculus of Variations. Indeed, if we were dealing with a standard constrained problem, the solution would coincide with the solution of the problem:

$$
\operatorname{Min}_{v \in X} J(v), \quad \text { such that } \quad v^{\prime}(0)=0, \quad \lim _{x \rightarrow+\infty} v(x)=0, \quad \int_{0}^{+\infty} G(v) d x=\frac{M}{2}
$$

where

$$
J(v)=\frac{1}{2} \int_{0}^{\zeta(t)}\left|v_{x}\right|^{2} d x-\frac{1}{2} \int_{0}^{\zeta(t)}|v|^{2} d x, \quad \text { and } \quad G(v)=|v|
$$

Then, by using the theory of Lagrange multipliers we would have that $v$ would solve the unconstrained minimization problem: 


$$
\operatorname{Min}_{v \in X} J(v)+\lambda \int_{0}^{+\infty} G^{\prime}(v) d x, \quad \text { such that } \quad v^{\prime}(0)=0, \quad \lim _{x \rightarrow+\infty} v(x)=0
$$

for some constant $\lambda$, where $G^{\prime}(v)=\operatorname{sign}(v)= \pm 1$ depending on the (positive or negative) sign of $v$. Thus, $v$ would be the solution of the associate Euler-Lagrange optimality equation

$$
-v_{x x}-v=\lambda G^{\prime}(v)
$$

and so, on the set where $v>0$ we would have $-v_{x x}-v=\lambda$, which is not the equation stated in the original formulation, i.e., $-v_{x x}-v=0$. Then, we derive the conclusion that the equation is not verified in the whole halfline $(0,+\infty)$ and that the integral constraint must be carefully considered.

We recall that here we look for a continuous nonnegative function $v \geq 0$ and that the differential equation must be verified at the interior of the set $\{v>0\}:=$ $\{x \in(0,+\infty): v(x)>0\}$. Since the solutions of the ODE, $v_{x x}+v=0$, are explicitly given by $v(x)=A \cos x+B \sin x$, we see that none of them can satisfy $v_{x}\left(\zeta_{\infty}\right)=0$ if $\{v>0\}=\left(0, \zeta_{\infty}\right)$. So, necessarily, the $\operatorname{limit} \lim _{x / \zeta_{\infty}} v_{x}(x)$ is strictly negative (since the function is passing from positive values to zero). Moreover, if we extend $v$ by zero to the rest of $(0,+\infty)$ (as in FowLER et al., 2007) we obtain that $v_{x}(x)$ has a discontinuity at $x=$ $\zeta_{\infty}$. In particular, $v_{x x}$ is not an integrable function on $(0,+\infty)$ but a measure with a nonzero singular part.

This introduces our formulation of the constraint by means of the "measure"

$$
\mu=-v_{x x} \in \mathcal{M}(0,+\infty),
$$

where $\mathcal{M}(0,+\infty)$ is the space of Radon measures (see, for instance, Evans and GARIEPY, 1992). In fact, from the identity $v_{x x}=-v$ on $\{v>0\}$ we see that the (signed) Jordan decomposition of $\mu$ (in the form $\mu=\mu_{+}-\mu_{-}$, with $\mu_{+} \perp_{-}$) is given by

$$
\mu_{+}=v\left(\text { which is in } L^{1}(0, \infty)\right) \text { and } \mu_{-}=-c \delta_{\zeta_{\infty}} \text { for some } c>0,
$$

where $\delta_{\zeta_{\infty}}$ is the Dirac delta distribution located at interface $\zeta_{\infty} \in \mathbb{R}^{+}$, i.e., where $v\left(\zeta_{\infty}\right)=0$ (sometimes we shall use the alternative notation $\delta_{\zeta_{\infty}}=\delta_{\partial\{v=0\}}$ ). Thus, $\mu_{-}$is a singular measure with respect to the Lebesgue measure.

Moreover, we see that in other problems in which $v_{x}\left(\zeta_{\infty}\right)=0$, i.e., when the flux is continuous at the free boundary, we obtain

$$
\int_{0}^{+\infty} d \mu=-\int_{0}^{+\infty} v_{x x} d x=0
$$

since $v_{x}(0)=0$ and $v_{x}(x)=0$ if $x>\zeta_{\infty}$. In our case the relation (2.15) is equivalent to the constraint $\int_{0}^{+\infty} v d x=M / 2$ even if $v_{x}\left(\zeta_{\infty}\right)<0$. Indeed, as mentioned above we know that

$$
-v_{x x}-v=c \delta_{\partial\{v=0\}},
$$


which, in this stationary case, allows us to compute $c$ explicitly

$$
0=\int_{0}^{+\infty} d \mu=\int_{0}^{+\infty} v d x-\int_{0}^{+\infty} d \mu_{-}=\frac{M}{2}-c\left\langle\delta_{\zeta_{\infty}}, 1\right\rangle=\frac{M}{2}-c,
$$

i.e., $c=M / 2$ and thus, necessarily, $\mu_{-}=-M / 2 \delta_{\zeta_{\infty}}$. Moreover, integrating in (2.16) we have:

$$
0=\int_{0}^{+\infty} d \mu=-\int_{0}^{\zeta_{\infty}} v_{x x} d x+\int_{\zeta_{\infty}}^{+\infty} d \mu_{-}=-v_{x}\left(\zeta_{\infty}\right)-\frac{M}{2}
$$

and we deduce that, in the stationary case, the flux is determined by the integral constraint $\left(-v_{x}\left(\zeta_{\infty}\right)=M / 2\right)$ and reciprocally. Notice that, despite of $(2.15)$, we have

$$
\|\mu\|_{\mathcal{M}(0,+\infty)}=\left\|\mu_{+}\right\|_{\mathcal{M}(0,+\infty)}+\left\|\mu_{-}\right\|_{\mathcal{M}(0,+\infty)}=\int_{0}^{+\infty} v d x+\frac{M}{2}=M .
$$

The (symmetric) global formulation can be stated in the following terms: Find a stationary state $v(x)$ and a point $\zeta_{\infty} \in \mathbb{R}^{+}$satisfying

$$
(S P) \begin{cases}v_{x x}+v=(M / 2) \delta_{\zeta_{\infty}}, & \text { in } \mathcal{D}^{\prime}(0,+\infty), \\ v(x)>0, & x \in\left[0, \zeta_{\infty}\right), \\ v(x) \equiv 0, & x \geq \zeta_{\infty}, \\ v_{x}(0)=0 . & \end{cases}
$$

We have

Proposition 1. Given $M>0$ there exists a unique solution $\left(v(x), \zeta_{\infty}\right)$ of $(S P)$ given by

$$
\zeta_{\infty}=\frac{\pi}{2} \quad \text { and } \quad v(x)=\frac{M}{2} \cos x\left[1-H\left(x-\frac{\pi}{2}\right)\right],
$$

where $H(x-\pi / 2)$ denotes the Heaviside function located at $\pi / 2$ i.e.,

$$
v(x)= \begin{cases}(M / 2) \cos x & \text { if } x \in[0, \pi / 2] \\ 0 & \text { if } x \in(\pi / 2,+\infty) .\end{cases}
$$

Proof. We shall use a shooting argument. Obviously any solution of $(S P)$ must satisfy $v(0)>0$. Let $\alpha, \xi>0$ be positive parameters. By using the Laplace transform we can solve the initial value problem

$$
\left\{\begin{array}{l}
v^{\prime \prime}+v=(M / 2) \delta_{\xi}, \quad \text { in } \mathcal{D}^{\prime}(0,+\infty) \\
v(0)=\alpha \\
v^{\prime}(0)=0
\end{array}\right.
$$

so proving the uniqueness and the extension to $(0,+\infty)$ of the local solution of the stationary strong-local formulation. Indeed, if we denote by 


$$
Y(s)=L[v](s)=\int_{0}^{\infty} e^{-s x} v(x) d x
$$

the Laplace transform of $v$, we get that $L\left[v^{\prime \prime}\right]+L[v]=\frac{M}{2} L\left[\delta_{\xi}\right]$, and so

$$
s^{2} Y(s)-s v(0)-v^{\prime}(0)+Y(s)=\frac{M}{2} e^{-\xi s} .
$$

Using the initial conditions this becomes

$$
s^{2} Y(s)-s \alpha+Y(s)=\frac{M}{2} e^{-\xi} s,
$$

whence

$$
Y(s)=\alpha\left(\frac{s}{s^{2}+1}\right)+\frac{M}{2}\left(\frac{e^{-\xi s}}{s^{2}+1}\right) .
$$

The inverse transform is direct:

$$
v(x)=\alpha \cos x+\frac{M}{2} H(x-\xi) \sin (x-\xi),
$$

where $H(x-\xi)$ denotes the Heaviside function located at $\xi$. The free boundary condition $v(\xi)=0$ leads to the characteristic equation $v(\xi)=\alpha \cos \xi=0$, of solutions $\xi_{n}=(n+1 / 2) \pi, n=0,1, \ldots$ and of corresponding eigenfunctions

$$
\begin{aligned}
v_{\alpha, n}(x) & =\alpha \cos x+\frac{M}{2} H\left(x-\left(n+\frac{1}{2}\right) \pi\right) \sin \left(x-\left(n+\frac{1}{2}\right) \pi\right) \\
& =\cos x\left[\alpha-(-1)^{n} \frac{M}{2} H\left(x-\left(n+\frac{1}{2}\right) \pi\right)\right]
\end{aligned}
$$

and the solutions $v_{\alpha, n}(x)$ have a compact support $[0, \xi]$, with $v_{\alpha}(x) \equiv 0$ for $x \notin[0, \xi]$ only if $\alpha=\alpha(n)=(-1)^{n}(M / 2)$. Notice that $n$ odd shall imply changing sign solutions. The requirement $v(x)>0$ on $\left[0, \xi_{n}\right]$, with $v \equiv 0$ for $x \notin\left[0, \xi_{n}\right]$ fixes the unique solution of the original stationary free boundary problem which corresponds to the value $n=0$ and so we get (2.17).

Remark 1. The function (2.17) was obtained in Fowlen et al. (2007), for the local/strong formulation (i.e., without any global formulation). As we shall see in the next subsection, the global formulation is especially useful for the parabolic case. In Figure 2 the steady-state solution (2.17) for the global formulation (SP) is represented. We showed that it can be characterized as the first eigenfunction of the free boundary problem, where $\zeta_{\infty}=\pi / 2\left(v(x)\right.$ has a compact and connected support). Its total mass, in $\mathbb{R}^{+}$, is $M / 2$. Notice the discontinuity of the flux at the free boundary $\zeta_{\infty}$ where a Dirac's delta is generated by the diffusion operator. 


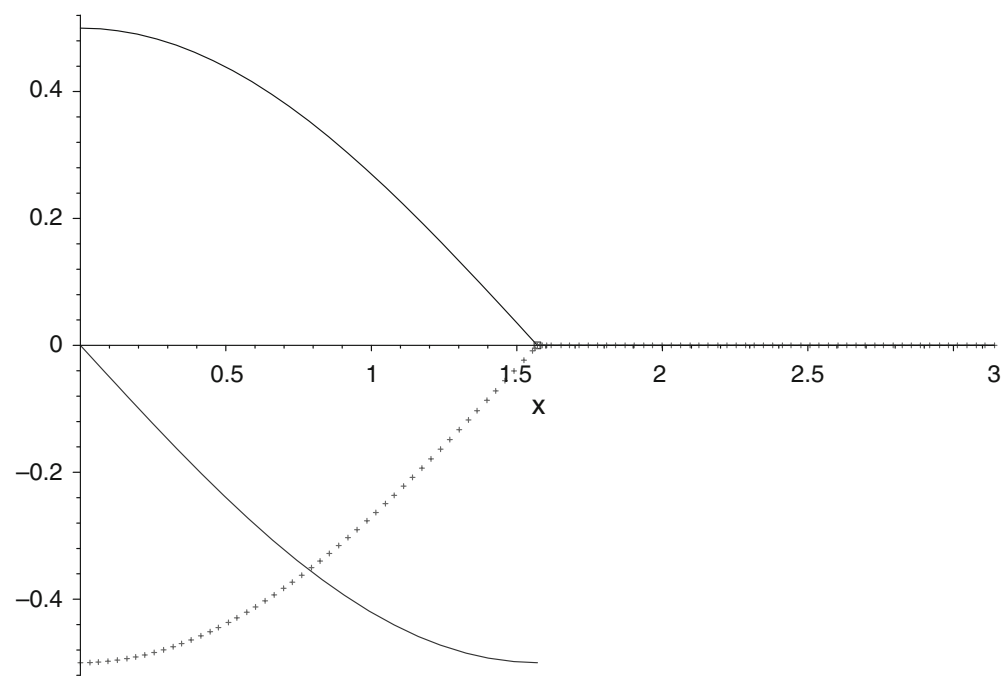

Figure 2

The (positive) stationary solution (2.17), its (negative and decreasing) derivative and the negative, increasing diffusive term $\left(v_{M / 2,0}^{m}\right)^{\prime \prime}(x)$ (dotted-line) for the global formulation (SP).

Remark 2. Problems of this type arise in fluid mechanics (problems of the Bernoulli type), in combustion and in plasma physics (see, e.g., DíAz et al., 2007, and references therein).

\subsection{Parabolic Case}

Given $T>0$ (arbitrary) and a continuous, symmetric, nonnegative initial data $u_{0}(x)$, with compact support $\left[0, \zeta^{0}\right]$ such that $\int_{0}^{+\infty} u_{0}^{m}(x) d x=M / 2$, we look for a continuous curve $\zeta:[0, T] \rightarrow \mathbb{R}^{+}$and a function $u: \mathbb{R}^{+} \times[0, T] \rightarrow[0,+\infty)$ such that $u$ satisfies the stronglocal formulation (SL). To prove their existence we shall use an auxiliary global formulation on the whole domain $\mathbb{R}^{+} \times[0, T]$. As in the stationary case, the global formulation of the partial differential equation includes a Dirac delta distribution located, for each $t \in(0, T]$, at the free boundary $x=\zeta(t)$ since the free boundary flux is discontinuous there (due to the mass constraint). To be precise we introduce the notation $\delta_{\partial\{u(t,)=0\}}$ to design the Dirac delta distribution located at the interface $x=\zeta(t)$ for each $t \in(0, T)$ (i.e., $\left.\delta_{\partial\{u(t, \cdot)=0\}}=\delta_{(\zeta(t), t)}\right)$. Nevertheless, the naturally associated problem

$$
\left(P_{0}\right)\left\{\begin{array}{lc}
u_{t}=\left(u^{m}\right)_{x x}+u^{m}-\frac{M}{2} \delta_{\partial\{u(t,)=0\}}, & \mathcal{D}^{\prime}\left(\mathbb{R}^{+} \times(0, T)\right), \\
u(x, 0)=u_{0}(x) & \text { a.e. } x \in\left(0, \zeta^{0}\right) \\
u(x, t)>0, & \text { a.e. }(x, t) \in \mathcal{P}_{T}, \\
u(x, t) \equiv 0, & \text { a.e. }(x, t) \notin \mathcal{P}_{T}, \\
u(\zeta(t), t)=0, \quad u_{x}(0, t)=0 & \text { a.e. } t \in(0, T), \\
\zeta(0)=\zeta^{0} \text { and } \zeta(t)>0 & \text { for any } t \in[0, T],
\end{array}\right.
$$


where $\mathcal{P}_{T}$ (the positivity subset of $u$ ) is defined by $\mathcal{P}_{T}=\left\{(x, t) \in \mathbb{R}^{+} \times[0, T]\right.$ : $0 \leq x<\zeta(t)\}$, has blow-up solutions. In fact, it is important to observe that the mere presence of the Dirac delta, in the parabolic case, does not prevent the occurrence of the blow-up phenomenon (well-known for the case of zero, continuous free boundary flux, see SAMARSKi et al., 1995, Chapter IV, Section 1.1). Indeed, the following result proves that it is possible to construct an infinite number of initial data such that the corresponding solutions $\left\{u_{T_{e}}\right\}$, with $T_{e}$ be a positive parameter, of problem $\left(P_{0}\right)$ (i.e., with a discontinuous free boundary flux condition) are not globally defined in time (the solution $u_{T_{e}}$ being defined on a finite time interval $\left[0, T_{e}\right)$ ). Moreover, $u_{T_{e}}$ verifies that

$$
\int_{0}^{+\infty} u^{m}(x, t) d x=\frac{M T_{e}^{1 /(m-1)}}{2\left(T_{e}-t\right)^{1 /(m-1)}} \quad \text { for } t \in\left[0, T_{e}\right) .
$$

Remark 3. There is a long list of references dealing with nonlinear parabolic equations involving similar Dirac delta distributions (see, e.g. CAFFARELLi et al., 1995, 1997, and their references) but the case of the simultaneous presence of the Dirac delta with a perturbation term of the form $u^{m}$ and with prescribed mass seems not to have been considered before. We also mention that some other problems formulated in terms of a quasi-linear parabolic equation with a constraint of total mass type were treated in NAZARET (2001).

Inspired by SAMARSKi et al. (1995) (see Chapter IV, Section 1.1), we search for some separable solutions, $u$, of the form

$$
u(x, t)=\left(T_{e}-t\right)^{-1 /(m-1)} \theta(x) .
$$

Then, by using the phase plane associated with the ordinary differential equation for $\theta(x)$ it is proved in DíAz et al. (in preparation), that the following result holds:

Proposition. i) For any $c>0$, the problem

$$
\begin{cases}w^{\prime \prime}+w-\frac{1}{m-1} w^{1 / m}=c \delta_{\zeta^{0}}, & \mathcal{D}^{\prime}(0,+\infty), \\ w(x)=0 & x>\zeta^{0}, \\ w^{\prime}(0)=0, & \end{cases}
$$

admits a unique nonnegative solution $w$ such that

$$
\int_{0}^{+\infty} w(x) d x=c .
$$

ii) If we take $\theta=w^{1 / m}$ and $c=(M / 2) T_{e}{ }^{m /(m-1)}$ then the pair $u_{T_{e}}(x, t)=\left(T_{e}-t\right)^{-1 /(m-1)}$ $\theta(x)$ and $\zeta(t) \equiv \zeta^{0}$ satisfies $\left(P_{0}\right)$ for $u_{0}(x):=T_{e}^{-1 /(m-1)} w^{1 / m}(x)$.

The above result explains that the reformulation of the mass constraint in terms of the solution of a global partial differential equation in the whole domain $\mathbb{R}^{+} \times(0, T)$ requires 
some additional condition (besides the presence of the mentioned Dirac delta at the equation). To do that, we start by noting that if we define (for a.e. $t \in(0, T)$ fixed) the spatial distribution

$$
\mu(t, \cdot):=u_{t}(t, \cdot)-\left(u^{m}\right)_{x x}(t, \cdot),
$$

then we must expect to know that, in fact, such a distribution is a bounded measure $\mathcal{M}(0,+\infty)$ (with compact support) since

$$
\mu(t, \cdot)=u^{m}(t, \cdot)-\frac{M}{2} \delta_{\partial\{u(t, \cdot)=0\}} .
$$

Moreover its signed (Jordan) decomposition, $\mu(t, \cdot)=\mu_{+}(t, \cdot)-\mu_{-}(t, \cdot)$, must be given by $\mu_{+}(t, \cdot)=u^{m}(t, \cdot)$ and $\mu_{-}(t, \cdot)=(M / 2) \delta_{\partial\{u(t, \cdot)=0\}}$. Now, as in the stationary case, we recognize that the mass constraint $\int_{0}^{+\infty} u^{m}(x, t) d x=M / 2$ is equivalent to the "zero total measure" condition

$$
\int_{0}^{+\infty} d \mu(t, \cdot)=0, \quad \text { for a.e. } t \in(0, T) .
$$

So, we arrive at the global formulation: Find a nonnegative function $u: \mathbb{R}^{+} \times[0, T) \rightarrow$ $[0,+\infty)$ such that

$$
(P) \begin{cases}u_{t}=\left(u^{m}\right)_{x x}+u^{m}-\frac{M}{2} \delta_{\partial\{u(t, \cdot)=0\}}, & \mathcal{D}^{\prime}\left(\mathbb{R}^{+} \times(0, T)\right), \\ u(x, 0)=u_{0}(x) & \text { a.e. } x \in(0,+\infty), \\ u_{x}(0, t)=0, u(x, t) \rightarrow 0 \text { as } x \rightarrow+\infty & \text { a.e. } t \in(0, T), \\ \mu(t, \cdot):=u_{t}(t, \cdot)-\left(u^{m}\right)_{x x}(t, \cdot) \text { satisfies }(2.19) & \text { a.e. } t \in(0, T) .\end{cases}
$$

Notice that now the compact support condition is not explicitly required. In fact, following the numerical experiences of FowLER et al. (2007), we conjecture that problem $(P)$ can be solved for suitable, strictly positive initial data $u_{0}(x)$ such that $u_{0}(x) \rightarrow 0$ as $x \rightarrow+\infty$. Notice also that if a solution $u$ of $(P)$ gives rise to a free boundary $\zeta$ $(t):=\partial\{u(t, \cdot)=0\}$ then, the zero total measure condition (2.19) implies that the free boundary flux must be given by

$$
-\left(u^{m}\right)_{x}(\zeta(t), t)=\frac{M}{2}-\int_{0}^{\zeta(t)} u_{t}(x, t) d x \quad \text { a.e. } t \in(0,+\infty) .
$$

Here (and, in fact, also in (2.19)) there is a slight abuse of notation since, a priori, $u_{t}(x, t)$ (respectively $\mu(t, \cdot)$ ) does not need to be a $L^{1}\left(\mathbb{R}^{+}\right)$function, but merely a bounded measure. Nevertheless we keep the classical notation for simplicity reasons. In any case, we see that in the transient regime the boundary flux at the free boundary is unknown (being also discontinuous), as opposed to the stationary case in which the flux (also discontinuous) can be explicitly known. Moreover, the above considerations allow us to conclude that any solution of the strong-local formulation (SL) solves problem $(P)$ and that any (regular enough) solution of $(P)$ with compact support satisfies the strong-local 
formulation (SL). In order to show the existence of a global solution $u$ of problem $(P)$ we use a two-step iterative approximation. The main idea is to construct $\left\{u_{2 n+1}: n=0\right.$, $1,2 \ldots\}$ as solutions of the problem with a semi-implicit linear source term

$$
\left(P_{2 n+1}\right):= \begin{cases}\left(u_{2 n+1}\right)_{t}=\left(\left(u_{2 n+1}\right)^{m}\right)_{x x}+\left(u_{2 n}\right)^{m-1}\left(u_{2 n+1}\right)-\frac{M}{2} \delta_{\partial\left\{\left(u_{2 n+1}\right)(t, \cdot)=0\right\}}, & \mathcal{D}^{\prime}\left(\mathbb{R}^{+} \times(0, T)\right), \\ \left(u_{2 n+1}\right)(x, 0)=u_{0}(x) & \text { a.e. } x \in(0,+\infty), \\ \left(u_{2 n+1}\right)_{x}(0, t)=0,\left(u_{2 n+1}\right)(x, t) \rightarrow 0 \text { as } x \rightarrow+\infty & \text { a.e. } t \in(0, T),\end{cases}
$$

(where for $n=0$ we use as $u_{2 n}$ the initial condition $u_{0}$ ) and then to construct the sequence $\left\{u_{2 n}: n=1,2 \ldots\right\}$ by requiring that

$\left(P_{2 n}\right):= \begin{cases}u_{2 n}(x, t)=C_{2 n}(t) u_{2 n-1}(x, t) & \text { for a.e. }(x, t) \in \mathbb{R}^{+} \times(0, T), \\ \int_{0}^{+\infty}\left(\left(u_{2 n}(x, t)\right)^{m-1}\left(u_{2 n-1}(x, t)\right) d x=\frac{M}{2}\right. & \text { for a.e. } t \in(0, T),\end{cases}$

for some $C_{2 n}(t)>0$.

The detailed proof of the convergence of the algorithm (contained in DíAz et al. (in preparation)) is quite technical and will not be presented here. For instance, many of the a priori estimates on $u_{2 n+1}$ were obtained previously for the solutions $u_{2 n+1, \varepsilon}$ of the equation obtained by replacing the singular equation of $\left(P_{2 n+1}\right)$ by the more regular equation

$$
\left(u_{2 n+1, \varepsilon}\right)_{t}=\left(\left(u_{2 n+1, \varepsilon}\right)^{m}\right)_{x x}+\left(u_{2 n}\right)^{m-1}\left(u_{2 n+1, \varepsilon}\right)-\beta_{\varepsilon}\left(u_{2 n+1, \varepsilon}\right),
$$

where $\beta_{\varepsilon}(r)$ is a regular nonnegative and bounded function, approximating $M / 2$ times the Dirac delta. Moreover, it is not difficult to show that

$$
u_{2 n+1}(x, t) \geq U(x, t) \text { for any } n \text { and a.e. }(x, t) \in \mathbb{R}^{+} \times(0, T),
$$

where $U(x, t)$ is the (bounded) solution of

$$
\left\{\begin{array}{lc}
U_{t}=\left(U^{m}\right)_{x x}-\frac{M}{2} \delta_{\partial\{U(t, \cdot)=0\}}, & \mathcal{D}^{\prime}\left(\mathbb{R}^{+} \times(0, T)\right), \\
U(x, 0)=u_{0}(x) & \text { a.e. } x \in(0,+\infty), \\
U_{x}(0, t)=0, U(x, t) \rightarrow 0=\text { as } x \rightarrow+\infty & \text { a.e. } t \in(0, T),
\end{array}\right.
$$

obtained by approximating the equation

$$
U_{\varepsilon t}=\left(\left(U_{\varepsilon}\right)^{m}\right)_{x x}-\beta_{\varepsilon}\left(U_{\varepsilon}\right),
$$

and passing to the limit as $\varepsilon \rightarrow 0$. Indeed, it is enough to apply the comparison principle (recall that $\left(u_{2 n}\right)^{m-1}\left(u_{2 n+1}\right) \geq 0$ ) to the approximate solutions to show that $u_{2 n+1, \varepsilon} \geq U_{\varepsilon}$ on $\mathbb{R}^{+} \times(0, T)$ and then, passing to the limit, we obtain (2.21). Function $U$ is bounded since if we introduce the function $V$, solution of the unperturbed problem

$$
\left\{\begin{array}{lc}
V_{t}=\left(V^{m}\right)_{x x} & \mathcal{D}^{\prime}\left(\mathbb{R}^{+} \times(0, T)\right), \\
V(x, 0)=u_{0}(x) & \text { a.e. } x \in(0,+\infty), \\
V_{x}(0, t)=0, V(x, t) \rightarrow 0 \text { as } x \rightarrow+\infty & \text { a.e. } t \in(0, T),
\end{array}\right.
$$


then we know that

$$
0 \leq U(x, t) \leq V(x, t) \quad \text { for any } t \in[0, T] \text { and a.e. } x \in \mathbb{R}^{+} .
$$

Indeed, it is enough to multiply the difference of the equations verified by $U$ and $V$, by an approximation of the test function $\operatorname{sign}_{+}(V-U)$ and use that $\frac{M}{2} \delta_{\partial\{U(t, \cdot)=0\}}$ $\operatorname{sign}_{+}(V-U) \leq 0$. On the other hand, by applying the results of BENILAN (1978), we derive

$$
0 \leq U(x, t) \leq V(x, t) \leq C \frac{\left\|u_{0}\right\|_{L^{1}\left(\mathbb{R}^{+}\right)}^{2 /(m+1)}}{t^{1 /(m+1)}} \text { for any } t \in[0, T] \text { and a.e. } x \in \mathbb{R}^{+} .
$$

Note that we can write that $\left\{u_{2 n+1}\right\}$ is a set of mild solutions satisfying

$$
\left\{\begin{array}{lc}
\left(u_{2 n+1}\right)_{t}-\left(\left(u_{2 n+1}\right)^{m}\right)_{x x}=f_{2 n+1}(t, x) & \mathcal{D}^{\prime}\left(\mathbb{R}^{+} \times(0, T)\right), \\
\left(u_{2 n+1}\right)(x, 0)=u_{0}(x) & \text { a.e. } x \in(0,+\infty), \\
\left(u_{2 n+1}\right)_{x}(0, t)=0,\left(u_{2 n+1}\right)(x, t) \rightarrow 0 \text { as } x \rightarrow+\infty & \text { a.e. } t \in(0, T),
\end{array}\right.
$$

with $f_{2 n+1}$ uniformly integrable in $\mathcal{M}(0,+\infty)$ in the sense that

$$
\left\|f_{2 n+1}\right\|_{L^{1}((0, T): \mathcal{M}(0,+\infty))} \leq K \quad \text { for any } n \text {, for some } K>0 .
$$

Then by a variation of the main result of Díaz and VRABIE (1989) (see also p. 70 of VRABIE 1987: both results concerning the special case in which $f_{2 n+1}$ is uniformly integrable in $L^{1}(0,+\infty)$ ), we obtain that $\left\{u_{2 n+1}\right\}$ is a relatively compact set of $C\left([0, T]: L^{1}\left(\mathbb{R}^{+}\right)\right)$. Then there exists a subsequence strongly convergent $\left\{u_{2 n+1}\right\} \rightarrow u$ in $C\left([0, T]: L^{1}\left(\mathbb{R}^{+}\right)\right)$. This, and the special construction of $\left\{u_{2 n}(x, t)\right\}$, implies the a priori estimate

$$
0<C_{2 n}(t) \leq\left[\frac{M}{2 \int_{0}^{+\infty}(U(x, t))^{m} d x}\right]^{1 /(m-1)} \quad \text { for a.e. } t \in(0, T)
$$

Moreover, at least under a physically natural assumption on the initial datum $u_{0}(x)$, we can ensure that $C_{2 n}(t)$ is uniformly bounded from above by a positive function for each $t \in$ $[0, T]$ (notice that we do not have such conclusion in the trivial case of $u_{0}(x) \equiv 0$ ). Indeed, let us assume that

$$
u_{0}(x)>0 \text { for any } x \in[0, \zeta(0)) .
$$

Then, as function $U(t,$.$) satisfies U_{t}(t,)=.\left(U^{m}(t, .)\right)_{x x}$ on $(0, \zeta(t))$, and $m>1$, we can apply a well-known local result (see KaLAshnikov, 1987) showing that if $U\left(0, x_{1}\right)>0$ then $U\left(t, x_{1}\right)>0$ for any $t>0$. In particular, we get that $U(t, x)>0$, for any $x \in[0, \zeta(0))$ and for any $t>0$. Thus, $\int_{0}^{+\infty}(U(x, t))^{m} d x>0$ for any $t>0$ and $0<C_{2 n}(t)$. This shows that $\left\{C_{2 n}(t)\right\}$ is uniformly bounded (and uniformly far from zero) in $L^{\infty}(0, T)$ and so, there exists $C^{*}(t)$ such that $C_{2 n}(.) \rightarrow C^{*}($.$) weakly-* in L^{\infty}(0, T)$. But, as $\left\{u_{2 n+1}\right\} \rightarrow u$ strongly in $C\left([0, T]: L^{1}\left(\mathbb{R}^{+}\right)\right)$we obtain: 


$$
\lim _{n \rightarrow \infty} u_{2 n}(x, t)=\lim _{n \rightarrow \infty}\left(C_{2 n}(t) u_{2 n-1}(x, t)\right)=\lim _{n \rightarrow \infty} C_{2 n}(t) \lim _{n \rightarrow \infty} u_{2 n-1}(x, t)=C^{*}(t) u(x, t) .
$$

Moreover, we can read the algorithm as

$$
\left(u_{2 n+1}\right)_{t}=\left(\left(u_{2 n+1}\right)^{m}\right)_{x x}+C_{2 n}(t)^{m-1}\left(u_{2 n-1}\right)^{m-1}\left(u_{2 n+1}\right)-\frac{M}{2} \delta_{\partial\left\{\left(u_{2 n+1}\right)(t, \cdot)=0\right\}},
$$

and so we get (by the Lebesgue's dominated convergence theorem) that $\left(u_{2 n-1}\right)^{m-1}$ $\left(u_{2 n+1}\right) \rightarrow u^{m}$ in $L^{1}\left(\mathbb{R}^{+} \times(0, T)\right)$.

In conclusion, we have shown

Theorem. Assume that $u_{0}(x)$ satisfies (2.22), then, there exists a function $C^{*}(t)>0$, $C^{*} \in L^{\infty}(0, T)$ and a function $u \in C\left([0, T]: L^{1}\left(\mathbb{R}^{+}\right)\right)$such that

$$
\left\{\begin{array}{lc}
u_{t}=\left(u^{m}\right)_{x x}+C^{*}(t)^{m-1} u^{m}-\frac{M}{2} \delta_{\partial\{u(t, \cdot)=0\},} & \mathcal{D}^{\prime}\left(\mathbb{R}^{+} \times(0, T)\right), \\
u(x, 0)=u_{0}(x) & \text { a.e. } x \in(0,+\infty), \\
u_{x}(0, t)=0, u(x, t) \rightarrow 0 \text { as } x \rightarrow+\infty & \text { a.e. } t \in(0, T),
\end{array}\right.
$$

and

$$
C^{*}(t)^{m-1} \int_{0}^{+\infty} u(x, t)^{m} d x=\frac{M}{2} .
$$

Remark. We do not know if $C^{*}(t)=1$, but by a rescaling $\widetilde{t}=k(t), y=Y(t, x)$ and $V=V(u, y, \widetilde{t})$ it is possible to reformulate the above equation in the terms

$$
V_{t}^{\sim}=\left(V^{m}\right)_{y y}+V^{m}-\frac{M}{2} \delta_{\partial\{V(\widetilde{t}, \cdot)=0\}} .
$$

\section{Numerical Results}

This section briefly describes the different techniques we used in the numerical resolution of the problem $(P)$. For each initial condition $h_{0}$, we compute its mass, say $M / 2$, and the associated stationary solution $v(x)$ given in (2.17) to which the solution should converge when $t \rightarrow+\infty$, (see Fowlen et al., 2007). As observed before, (P) is a free boundary problem with a non-local integral term and a Dirac's delta distribution in the absorption term. These characteristic features, along with the (weak) nature of the problem, suggest the development of the numerical resolution of the problem in the framework of finite elements. We first consider a time marching scheme in the coordinate $t$, of step $d t$. At each level in the time discretization, we shall employ a semi-implicit scheme in order to 
deal with the nonlinearities. An iterative (splitting) numerical scheme is implemented in order to impose the mass conservation constraint. In order to discretize with respect to the coordinate $x$, at each time level $l \cdot d t$, we will employ piecewise linear finite elements $L_{l, k}:=\left\{\phi \in C^{0}([0,+\infty)): \phi \mid E \in \mathbf{P}_{1}, \forall E \in \mathbf{T}_{l, k}\right\}$ in a uniform grid, $\mathbf{T}_{l, k}$, of step $k$. Also, $\mathbf{B}_{l, k}:=\left\{\phi_{i}\right\}$ is a base of finite linear elements in $L_{l, k}$.

Then, the discretized problem is formulated as follows:

Find $\left(u_{l+1}\right)_{k} \in L_{l, k},\left(u_{l+1}\right)_{k}=\sum_{j}\left(u_{l+1}\right)_{k}^{j} \phi_{j}$, such that

$$
\begin{aligned}
\int_{\mathbf{T}_{l, k}}\left(u_{l+1}\right)_{k} \phi_{i} d x= & \int_{\mathbf{T}_{l, k}}\left(u_{l}\right)_{k} \phi_{i} d x-\frac{3 d t}{2} \int_{\mathbf{T}_{l, k}}\left(u_{l}\right)_{k}^{\frac{1}{2}}\left(\left(u_{l+1}\right)_{k}\right)_{x} \phi_{i x} d x \\
& +d t \int_{\mathbf{T}_{l, k}}\left(u_{l+1}\right)_{k}^{\frac{3}{2}} \phi_{i} d x-d t \int_{\mathbf{T}_{l, k}} \frac{M}{2} \delta\left(u_{l}\right) \phi_{i} d x, \forall \phi_{i} \in \mathbf{B}_{\mathbf{l}, \mathbf{k}} .
\end{aligned}
$$

Note that $\delta$ is evaluated at the boundary obtained in the previous time step. In order to deal with the nonlinearities present in (3.23), as above stated and taking into consideration the mass conservation constraint, we shall consider the following iterative scheme: for $p=2 n+1$ from 1 to $N, n=0,1,2 \ldots$, and $N$ an odd number to be fixed, we consider the problem

$$
\begin{aligned}
\int_{\mathbf{T}_{l, k}}\left(u_{l+1,2 n+1}\right)_{k} \phi_{i} d x= & \int_{\mathbf{T}_{l, k}}\left(u_{l}\right)_{k} \phi_{i} d x-\frac{3 d t}{2} \int_{\mathbf{T}_{l, k}}\left(u_{l+1,2 n}\right)_{k}^{\frac{1}{2}}\left(\left(u_{l+1,2 n+1}\right)_{k}\right)_{x} \phi_{i x} d x \\
& +d t \int_{\mathbf{T}_{l, k}}\left(u_{l+1,2 n}\right)_{k}^{\frac{1}{2}}\left(u_{l+1,2 n+1}\right)_{k} \phi_{i} d x-d t \int_{\mathbf{T}_{l, k}} \frac{M}{2} \delta\left(u_{l}\right) \phi_{i} d x, \forall \phi_{i} \in \mathbf{B}_{\mathbf{l}, \mathbf{k}},
\end{aligned}
$$

where $\left(u_{l+1,2 n}\right)_{k}$ has been rescaled before being introduced in (3.23) so that $\int\left(u_{l+1,2 n}\right) k^{3 / 2}=M / 2$ according to $\left(\mathrm{P}_{2 n}\right)$, i.e., $\left(u_{l+1,2 n}\right)_{k}=C_{l+1,2 n}\left(u_{l+1,2 n-1}\right)_{k}$. The resulting system of equations for the nodal values at the $(2 n+1)$ th-step is solved with the Gauss-Seidel method. In order to initiate the iterative scheme, one can take as $\left(u_{l+1, p=1}\right)_{k}$ the values obtained in the previous time step, that is to say, $\left(u_{l+1, p=1}\right)_{k}=u_{l}$. The scheme finishes assuming the values for the $(l+1)$-time level given by $u_{l+1}=\left(u_{l+1, p=N}\right)_{k}$.

In Figure 3 we present the numerical results obtained considering as an initial condition the function $u_{0}(x)=1-x^{2}$, where we have plotted the graphics obtained for different steps in time. The results reveal the convergence, as expected, to the support of the stationary solution in a monotonic way. We also can see how the maximum of the solution does not blow up (by the contrary, it is decreasing in time in this special case).

In Figures 4 and 5, we present 3-D graphics of the results corresponding to $u(x, t)$ and $-u(x, t)$, respectively. We also present two tables in which we show results concerning the values of the constant of the scaling at the final step $N$ and the location of the boundary for different levels in time. 


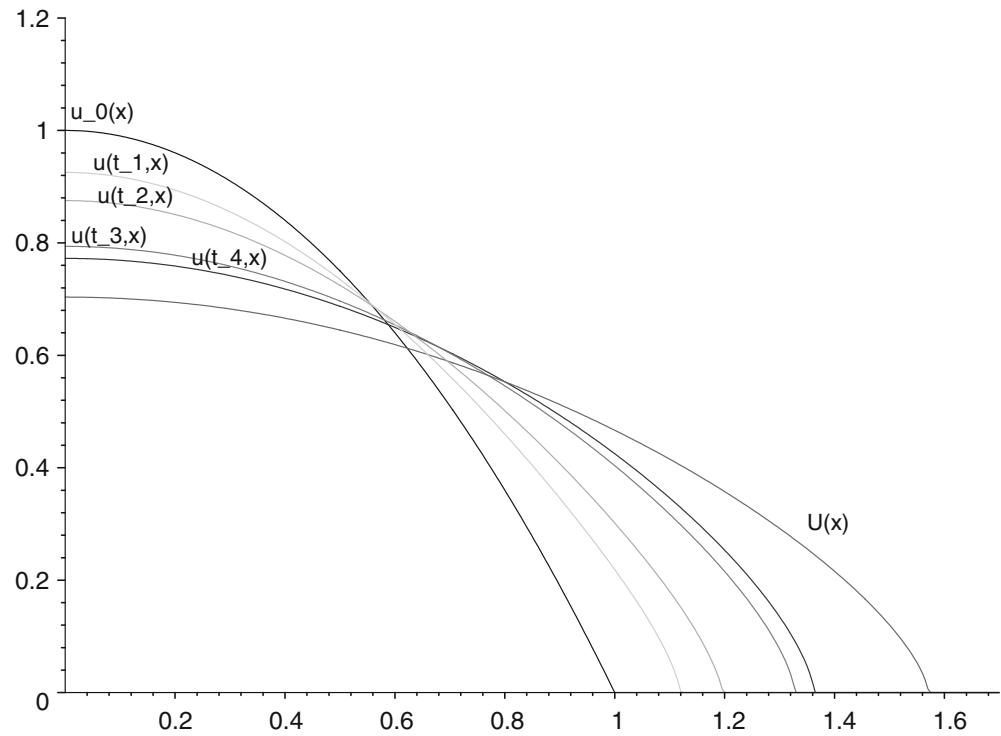

Figure 3

Numerical results obtained for $u(x, t)$ for different time levels. The initial condition is labelled by $u_{0}$ and the stationary solution by $u$.

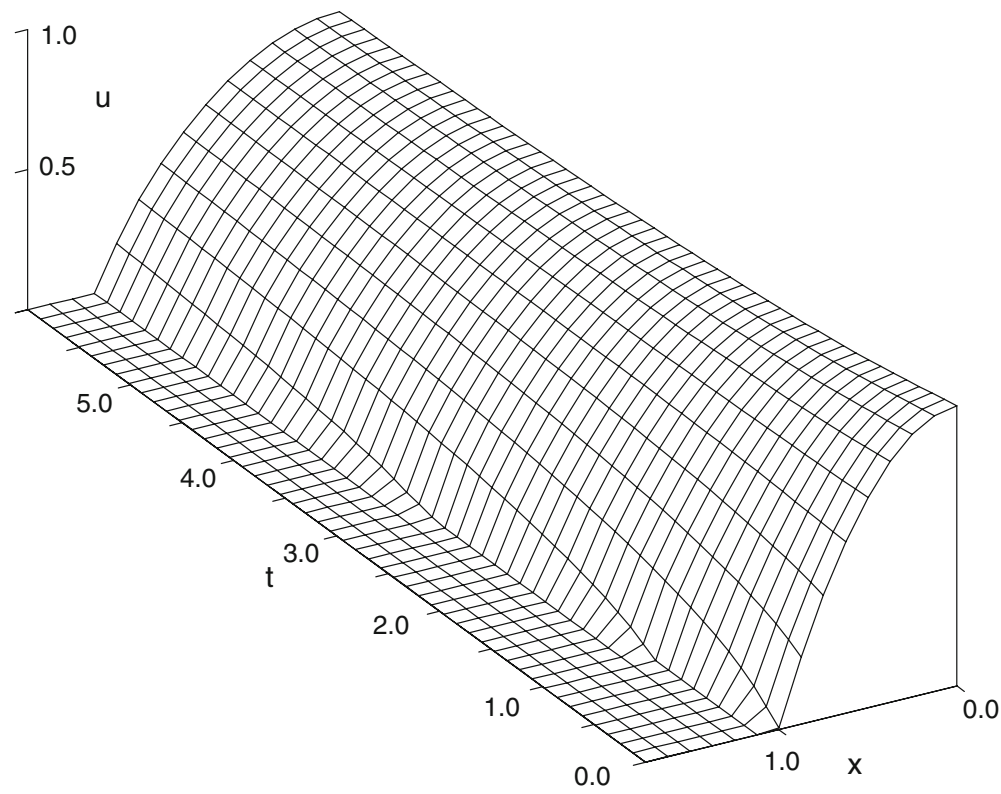

Figure 4

3-D representation of the numerical results obtained for $u(x, t)$. 


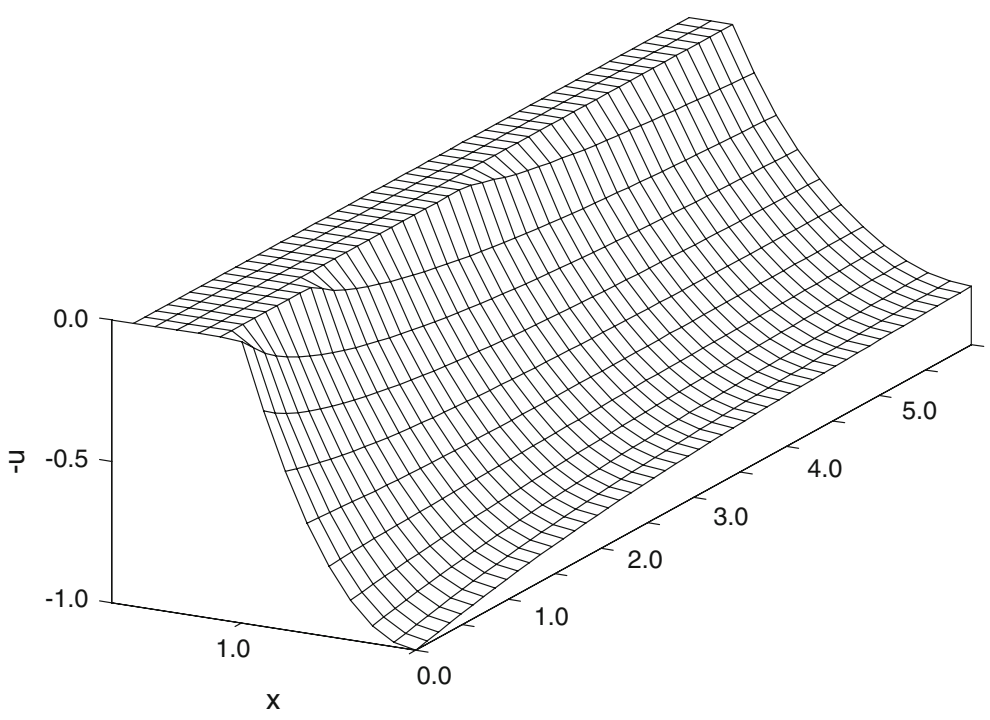

Figure 5

3-D representation of the numerical results obtained for $-u(x, t)$.

\subsection{Summary and Conclusions}

A coupled model describing the evolution of the topographic elevation and the depth of the overland water film is studied here when considering the overland flow of water over an erodible sediment. The instability of the spatially uniform solution corresponds to the formation of rills, which in reality then grow and coalesce to form large-scale river channels.

We started by considering the deduction and mathematical analysis of a deterministic model describing river channel formation and the evolution of its depth. We complete the previous modeling of the problems by SMITH and BRETHERTON (1972) and FOWLER et al. (2007), obtaining a model which involves a degenerate nonlinear parabolic equation (satisfied on the interior of the support of the solution) with a superlinear source term and a prescribed constant mass. We propose here a global formulation of the problem (formulated in the whole space, beyond the support of the solution) which allows us to show the existence of a solution and leads to a suitable numerical scheme for its approximation. As we show, the solution does not blow up despite the presence of the superlinear forcing term at the equation thanks to the mass constraint.

A particular feature of the model for channel evolution which we have studied is that the degeneracy of the equation causes the channel width to be self-selecting. This is of some interest in the geomorphological literature, since the issue of channel width determination is one that has caused some difficulties (e.g., PARKER, 1978). 
Table 1

\begin{tabular}{lc}
\hline Time $(\mathrm{t})$ & Results for $C_{N}$ \\
\hline 1.0 & 1.00000071770833 \\
2.0 & 1.00000061614692 \\
3.0 & 1.00000056130953 \\
4.0 & 1.00000051022114 \\
5.0 & 1.00000050094979 \\
6.0 & 1.00000046809999 \\
\hline
\end{tabular}

Table 2

\begin{tabular}{lc}
\hline Time $(\mathrm{t})$ & $\begin{array}{c}\text { Location of the } \\
\text { boundary }(\mathrm{x})\end{array}$ \\
\hline 1.0 & 1.31999997049570 \\
2.0 & 1.36999996937811 \\
3.0 & 1.37499996926036 \\
4.0 & 1.38849999690422 \\
5.0 & 1.38999996893108 \\
6.0 & 1.39499996881932 \\
\hline
\end{tabular}

\section{Acknowledgments}

The research of the four authors was partially supported by the projects MTM200503463 of the DGISGPI (Spain). A. C. F. acknowledges the support of the Mathematics Applications Consortium for Science and Industry (http://www.macsi.ul.ie) funded by the Science Foundation Ireland mathematics initiative grant 06/MI/005. The research of J.I.D., A.I.M and E.S. was also supported by the project CCG07-UCM/ESP-2787 of the DGUIC of the CAM and the UCM.

\section{REFERENCES}

Benilan, P. (1978), Operateurs accretifs et semigroups dans les espaces $L^{p}$, Functional Analysis and Numerical Analysis, France-Japan Seminar (H. Fujita, ed.), Japan Society for the Promotion of Science, Tokio, pp. 1553.

Birnir, B., Smith, T. R., and Merchant, G. E. (2001), The scaling of fluvial landscapes. Comput. Geosci. 27, 1189-1216.

Caffarelli, L. A., Lederman, C., and Wolanski, N. (1997), Pointwise and viscosity solutions for the limit of a two-phase parabolic singular perturbation problem, Indiana Univ. Math. J. 46(3), 719-740.

Caffarelli, L. A. and Vázquez, J. L. (1995), A free-boundary problem for the heat equation arising in flame propagation, Trans. Amer. Math. Soc. 347(2), 411-441.

Díaz, J. I., Fowler, A. C., Muñoz, A. I., and Schiavi, E., Article in preparation.

Díaz, J. I., Padial, J. F., and Rakotoson, J. M. (2007), On some Bernouilli free boundary type problems for general elliptic operators, Proc. Roy. Soc. Edimburgh 137A, 895-911. 
Díaz, J. I. and VRabie, I. (1989), Proprietés de compacité de l'opérateur de Green généralisé pour l'équation des milieux poreux, Comptes Rendus Acad. Sciences, París 309, Série I, 221-223.

Evans, L. C. and Gariepy, R. F., Measure Theory and Fine Properties of Functions (Studies in Advanced Mathematics, CRC Press, Boca Raton, 1992).

Fowler, A. C., Kopteva, N., and OAKLEy, C. (2007), The formation of river channels, SIAM J. Appl. Math. 67, 1016-1040.

Howard, A. D. (1994), A detachment-limited model of drainage basin evolution, Water Resour. Res. 30, 2261-2285.

IzUMI, N. and PARKer, G. (1995), Inception and channellization and drainage basin formation: Upstream-driven theory, J. Fluid Mech. 283, 341-363.

IzUmi, N. and PARKer, G. (2000), Linear stability analysis of channel inception: Downstream-driven theory, J. Fluid Mech. 419, 239-262.

Kalashnikov, A. S. (1987), Some problems of the qualitative theory of second-order nonlinear degenerate parabolic equations, Uspekhi Mat. Nauk 42, 135-176.

Kramer, S. and Marder, M. (1992), Evolution of river networks, Phys. Rev. Lett. 68, 205-208.

Loewenherz, D. S. (1991), Stability and the initiation of channelized surface drainage: A reassessment of the short wavelength limit, J. Geophys. Res. 96, 8453-8464.

LOEWENHERZ-LAWRENCE, D. S. (1994), Hydrodynamic description for advective sediment transport processes and rill initiation, Water Resour. Res. 30, 3203-3212.

NAZARET, B. (2001), Heat flow for extremal functions in some subcritical Sobolev inequalities, Appl. Anal. 80, 95-105.

Meyer-Peter, E. and Müller, R. (1948), Formulas for bed-load transport, Proc. Int. Assoc. Hydraul. Res., 3rd Annual Conference, Stockholm, 39-64.

PARKer, G. (1978), Self-formed straight rivers with equilibrium banks and mobile bed, Part 1. The sand-silt river, J. Fluid Mech. 89, 109-125.

Samarski, A. A., Galaktionov, V. A., Kurdyumov, S. P., and Mikhailov, A. P., Blow-up in quasilinear parabolic equations (Walter de Gruyter, Berlin, 1995).

Smith, T. R., Birnir, B., and Merchant, G. E. (1997), Towards an elementary theory of drainage basin evolution: II. A computational evaluation, Comput. Geosci. 23, 823-849.

Smith, T. R. and Bretherton, F. P. (1972), Stability and the conservation of mass in drainage basin evolution, Water Resour. Res. 8, 11, 1506-1529.

Tucker, G. E. and Slingerland, R. L. (1994), Erosional dynamics, flexural isostasy, and long-lived escarpments: A numerical modeling study, J. Geophys. Res. 99, 12.229-12.243.

VRABIE, I. I., Compactness Methods for Nonlinear Evolutions (Pitman Longman, London, 1987).

Willgoose, G., Bras, R. L., and Rodríguez-Iturbe, I. (1991), A coupled channel network growth and hillslope evolution model: I. Theory, Water Resour. Res. 27, 1671-1684.

(Received September 19, 2007, revised July 17, 2008, accepted August 14, 2008)

Published Online First: October 29, 2008

To access this journal online:

www.birkhauser.ch/pageoph 